\title{
In Memory of Paul Bertelson (1926-2008)
}

\author{
Jean Vroomen · Beatrice de Gelder
}

Received: 21 April 2009/Accepted: 23 April 2009/Published online: 5 May 2009

(C) The Author(s) 2009. This article is published with open access at Springerlink.com

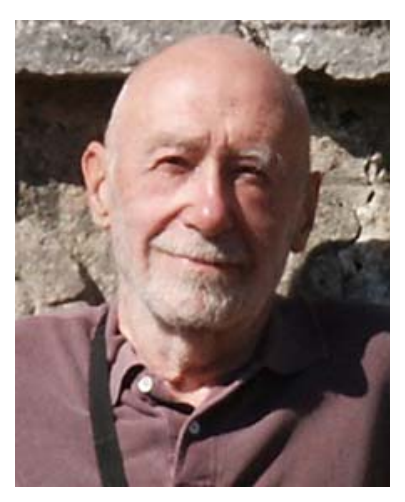

Here, we would like to pay tribute to Paul Bertelson who died on the 26th of November 2008. Paul was Professor Emeritus at the Université Libre de Bruxelles, Belgium and a visiting professor at Tilburg University, the Netherlands. Paul started his career as a student in Economics, but soon after receiving his degree he became interested in human performance and turned to psychology. As a young scientist, he visited the Applied Psychology Unit in Cambridge for a sabbatical year in 1957. During that stay, he carried out seminal work with Donald Broadbent on card sorting and reaction time, focussing on the issue of sequential dependencies. Paul was one of the first who conducted research in the then developing field of 'mental chronometry'. The basic notion was that mental processes could be studied through human reaction times, an idea that, in

This article is published as part of the Special Issue on Multisensory Integration.

J. Vroomen $(\bowtie) \cdot$ B. de Gelder

Tilburg University, Tilburg, The Netherlands

e-mail: j.vroomen@uvt.nl retrospect, turned out to be extremely valuable as it is still one of the fundaments that modern cognitive psychology is built upon.

In 1965, Paul went back to the Université Libre de Bruxelles where he became a full professor in Experimental Psychology and founded the Laboratory for Experimental Psychology. In the years that followed, he showed a broad interest in the working of the human mind. Under his scholarship, research was initiated in a variety of topics like the serial organization of action, the refractory period, time order perception, cerebral lateralization, perceptual asymmetries, spatial attention, speech and spoken word recognition, word recognition in reading, Braillereading, reading acquisition and phonology, and illiteracy.

Paul's contribution to the research area that would now be called 'multisensory perception' was extremely important. More than a century ago, von Helmholtz had already shown that the visual-motor system is remarkably flexible, as it adapts to shifts of the visual field induced by wedge prisms. As an example, if prism-wearing subjects had to pick up a visually displaced object, they would quickly adapt to the new sensory-motor arrangement and even after only a few trials, small visual displacements would go unnoticed. His good friend Richard Held developed the notion that active feedback from the motor system was required for such visual-motor adaptation to occur, as adaptation was reduced if subjects were being moved by an experimenter rather then if they moved themselves. Paul though showed that active feedback was not always necessary, as adaptation to spatial displacement could also be demonstrated in the audio-visual domain. Together with Monique Radeau, he reported that listeners hearing a sound while seeing a spatially-displayed flash had the (false) impression that the sound originated from the light. He named this phenomenon the 'ventriloquist 
illusion', as he considered it a stripped-down version of what a ventriloquist was doing when performing on stage. Typical of his way of thinking, Paul was concerned that the experimental situation used to induce the ventriloquist illusion might actually not measure truly perceptual effects, but rather just response biases. That is, a sound presented in synchrony with a flash might either shift the apparent location of the sound at a perceptual level or, in Paul's words, subjects may just be nice to the experimenter and point to the light rather than the sound. In both cases, responses would be shifted towards the light, but clearly, only the first explanation was interesting for him as he did not want to study social, but experimental psychology. As for the ventriloquist illusion, Paul succeeded in disentangling these alternatives by showing that if subjects were exposed to displaced sound-light stimuli for a prolonged time, there were adaptive shifts in unimodal sound localization as well (and thus in the absence of distracter lights). He discovered that the apparent location of a sound was shifted in the direction of a previously seen distracter light, and this 'ventriloquist aftereffect' was for him a particularly clear example that the shift of a ventriloquized sound occurred at a perceptual level, as opposed to participants being just 'nice' to him.

Another area Paul worked on in the mid-eighties and that became influential was reading acquisition. He was intrigued by the question why the acquisition of skills in reading and writing is so much more difficult than the earlier acquisition of speech communication. Basically, every child learns to listen and speak in the first years of life, but reading and writing is remarkably difficult as it requires formal tuition at a much later age. Moreover, despite formal training, a substantial minority of reading students will never learn to read at appropriate levels. The notion being developed at that time was that a fundamental difficulty might be that speech needs to be represented as a succession of units, and that acquiring such 'phonological awareness' might be the major difficulty that some children encounter. Phonological awareness was usually assessed by asking children to isolate or manipulate individual phonemic segments in words, an ability that develops around first grade. This improvement could in principle be ascribed to one of two factors (1) cognitive-linguistic skills that mature at about the age of six, independent of formal reading instruction or (2) as children learn about letter-sound correspondence when learning to read, they become aware that words are formed of the sounds that the letters represent. Paul made valuable contributions to this discussion by showing that a substantial part of the story could be attributed to the latter view. He showed, with Jose Morais,
Alain Content and Jesus Alegria, that adult Portugese illiterates who lacked formal tuition but who were otherwise normal, were poor in explicit speech segmentation tasks that required manipulations at the phonemic level. Thus, phonological awareness did not develop spontaneously, but required reading tuition in an alphabetic script.

After Paul's formal retirement in Brussels, he continued his scientific work mainly at Tilburg University with Beatrice de Gelder and Jean Vroomen. One of the multisensory themes they addressed was whether the processing of audiovisual speech differs in any sense from the processing of audiovisual spatial information. Ever since the work by McGurk and MacDonald, it was known that lipread information had profound effects on the way auditory speech was perceived. For example, if subjects hear /aba/ while seeing the face of a speaker saying /ada/, they report 'hearing' /aga/, a phoneme that is a fusion of the two information sources. One of the questions that was addressed was whether subjects would also adapt to audiovisual discordance in speech. From the ventriloquist aftereffect, it was known that the spatial discordance between the heard and seen position of an audiovisual event was reduced by shifting the least reliable information source (i.e., sound localization) towards the more reliable one (visual localization). Would the same occur for audiovisual speech? The answer was a clear 'yes': audiovisual discordance in speech was, as in space, reduced because lipread information could shift the interpretation of an ambiguous sound towards the non-ambiguous lipread phoneme, a phenomenon termed 'phonetic recalibration'.

Besides being an outstanding researcher, Paul was also an influential figure in European psychology throughout his career and was recognized as the leading continental psychologist since the sixties. He was one of the co-members who launched the European Society for Cognitive Psychology (ESCOP) in 1985. To honor his contributions to the field, the board of ESCOP decided to award a prestigious prize every two years to a young and outstanding scientist, naming the award the "Paul Bertelson award". For many who knew Paul, he will be remembered as an outstanding scholar, a generous colleague, and a truly engaging personality with his very own wonderful sense of humor. A presentation of his life and work can be found at www.PaulBertelson.com.

Open Access This article is distributed under the terms of the Creative Commons Attribution Noncommercial License which permits any noncommercial use, distribution, and reproduction in any medium, provided the original author(s) and source are credited. 